\title{
CURVES ON ALGEBRAIC MODELS OF SMOOTH MANIFOLDS
}

\author{
W. KUCHARZ
}

\section{Introduction}

Let $X$ be a compact nonsingular real algebraic set (in $\mathbb{R}^{n}$ for some $n$ ). In real algebraic geometry one frequently encounters the subgroup

$$
H_{k}^{\operatorname{alg}}(X, \mathbb{Z} / 2)
$$

of the homology group $H_{k}(X, \mathbb{Z} / 2)$, generated by the homology classes represented by irreducible algebraic subsets of $X$ of dimension $k$. The reader may refer to [4] for a short survey of properties and applications of the groups $H_{k}^{\text {alg }}(-, \mathbb{Z} / 2)$. Some homology classes are always in $H_{k}^{\text {alg }}(X, \mathbb{Z} / 2)$. Namely, if $w_{i}(X)$ is the $i$ th StiefelWhitney class of $X$, then the homology class Poincaré dual to the cup product $w_{i_{1}}(X) \cup \ldots \cup w_{i_{r}}(X)$, where $i_{1}+\cdots+i_{r}=\operatorname{dim} X-k$, is in $H_{k}^{\text {alg }}(X, \mathbb{Z} / 2)$. In general, it is very difficult to compute $H_{k}^{\text {alg }}(X, \mathbb{Z} / 2)$, even for $k=1$. This paper deals with certain interesting, also hard to compute, subgroups of $H_{1}^{\text {alg }}(X, \mathbb{Z} / 2)$.

By a real algebraic curve we mean a one-dimensional irreducible real algebraic set. If $C$ is such a curve, then there is a unique (up to isomorphism) nonsingular projective complex algebraic curve $V$ defined over $\mathbb{R}$, whose set of real points $V(\mathbb{R})$ is birationally equivalent to $C$. The genus of $C$ is, by definition, the genus of $V$. In particular, $C$ is rational (that is, birationally equivalent to $\mathbb{R}$ ) if and only if it is of genus 0 . The curve $C$ is said to be dividing if $V \backslash V(\mathbb{R})$ is disconnected.

Given a nonnegative integer $g$, denote by

$$
A(X, g)(\operatorname{resp} . B(X, g))
$$

the subgroup of $H_{1}^{\text {alg }}(X, \mathbb{Z} / 2)$ generated by the homology classes represented by algebraic curves in $X$ of genus $g$ (resp. of genus at most $g$ ). Obviously, $A(X, g)$ is a subgroup of $B(X, g)$. Moreover, $B(X, g)=H_{1}^{\text {alg }}(X, \mathbb{Z} / 2)$, provided $g$ is sufficiently large (depending on $X$ ).

We are also interested in the subgroup

$$
D(X)
$$

of $H_{1}^{\text {alg }}(X, \mathbb{Z} / 2)$ generated by the homology classes represented by dividing algebraic curves in $X$. Clearly, $A(X, 0)$ is a subgroup of $D(X)$.

Recall that every compact smooth (of class $C^{\infty}$ ) manifold $M$ is diffeomorphic to a nonsingular real algebraic set, called an algebraic model of $M$, cf. [11] or [3, Theorem 14.1.10] (and also [9] for a weaker but influential result). We precisely describe how the groups $A(X, g)$ and $B(X, g)$ vary as $X$ runs through the class of all algebraic models of $M$.

Received by the editors March 20, 2007. 
Theorem 1.1. Let $M$ be a compact connected smooth manifold of dimension at least 2 , and let $g$ be a nonnegative integer. For any subgroup $G$ of $H_{1}(M, \mathbb{Z} / 2)$, there exist an algebraic model $X$ of $M$ and a smooth diffeomorphism $\varphi: M \rightarrow X$ with

$$
\varphi_{*}(G)=A(X, g)=B(X, g) .
$$

Here, as usual,

$$
\varphi_{*}: H_{*}(M, \mathbb{Z} / 2) \longrightarrow H_{*}(X, \mathbb{Z} / 2)
$$

denotes the homomorphism induced by $\varphi$. Note that in Theorem 1.1 one cannot take $G=0$ and replace the groups $A(X, g)$ and $B(X, g)$ by $H_{1}^{\text {alg }}(X, \mathbb{Z} / 2)$. Indeed, if $X$ is any algebraic model of real projective $n$-space $\mathbb{P}^{n}(\mathbb{R})$, with $n$ even, then $H_{k}^{\text {alg }}(X, \mathbb{Z} / 2)=H_{k}(X, \mathbb{Z} / 2) \cong \mathbb{Z} / 2$ for $0 \leq k \leq n$ since $w_{1}(X) \neq 0$. Moreover, it is not known whether there is an algebraic model $X$ of the $n$-torus $\mathbb{R}^{n} / \mathbb{Z}^{n}$, with $n$ odd greater than 1 , for which $H_{1}^{\text {alg }}(X, \mathbb{Z} / 2)=0$.

We also have an analogous result to Theorem 1.1 dealing with the group $D(-)$.

Theorem 1.2. Let $M$ be a compact connected smooth manifold of dimension at least 2. Assume that $M$ is orientable and the group $H_{1}(M, \mathbb{Z})$ has no 2-torsion. For any subgroup $G$ of $H_{1}(M, \mathbb{Z} / 2)$, there exist an algebraic model $X$ of $M$ and a smooth diffeomorphism $\varphi: M \rightarrow X$ with

$$
\varphi_{*}(G)=A(X, 0)=D(X) .
$$

We were not able to determine whether the assumption that $M$ is orientable and $H_{1}(M, \mathbb{Z})$ has no 2 -torsion is necessary. Theorem 1.2 implies that, in general, $D(X) \neq$ $H_{1}^{\text {alg }}(X, \mathbb{Z} / 2)$. Indeed, there is an algebraic model $X$ of $\mathbb{P}^{2}(\mathbb{C}) \times(\mathbb{R} / \mathbb{Z})$, where $\mathbb{P}^{2}(\mathbb{C})$ is the complex projective plane, with $D(X)=0$. On the other hand, $w_{4}(X) \neq 0$ and hence $H_{1}^{\text {alg }}(X, \mathbb{Z} / 2)=H_{1}(X, \mathbb{Z} / 2) \cong \mathbb{Z} / 2$.

Both Theorems 1.1 and 1.2 are proved in the next section.

\section{Proofs}

A compact nonsingular real algebraic set $X$ is said to have totally algebraic homology if

$$
H_{k}^{\operatorname{alg}}(X, \mathbb{Z} / 2)=H_{k}(X, \mathbb{Z} / 2)
$$

for all $k \geq 0$. This notion will play an important role in our proofs.

Lemma 2.1. Let $g$ be a nonnegative integer. Any compact smooth manifold $N$ has an algebraic model $Y$ such that for every real algebraic curve $D$ of genus at most $g$, every rational map from $D$ into $Y$ is constant.

Proof. Let $A$ be a compact connected nonsingular real algebraic curve of genus at least $g+1$. Let $B=A \times \cdots \times A$ be the $q$-fold product, where $q \geq 2 \operatorname{dim} N+1$, and let $e: N \rightarrow B$ be a smooth embedding. Endow the space $C^{\infty}(N, B)$ of smooth maps from $N$ into $B$ with the $C^{\infty}$ topology and choose a neighborhood $V$ of $e$ in $C^{\infty}(N, B)$ consisting of smooth embeddings. Since $A$ is connected, it follows that $B$ has totally algebraic homology, and hence by [2, Theorem 3] or [1, Proposition 2.8], there exist an algebraic model $Y$ of $N$, a smooth diffeomorphism $\psi: N \rightarrow Y$, and a regular map $f: Y \rightarrow B$ such that $f \circ \psi$ is in $V$. In particular, $f$ is a smooth embedding, and consequently injective. 
Let $D$ be a real algebraic curve of genus at most $g$ and let $\rho: D \rightarrow Y$ be a rational map. Since the genus of $D$ is strictly less than the genus of $A$, the rational map $f \circ \rho: D \rightarrow B$ is constant in virtue of the Riemann-Hurwitz theorem. It follows that $\rho$ is constant, $f$ being injective. The proof is complete.

Denote by $\mathbb{G}_{r, s}(\mathbb{R})$ the Grassmannian of $s$-dimensional vector subspaces of $\mathbb{R}^{r}$. Recall that $\mathbb{G}_{r, s}(\mathbb{R})$ can be regarded in a natural way as an algebraic subset of $\mathbb{R}^{m}$ for some $m$, cf. [3, Theorem 3.4.4].

Lemma 2.2. Let $C_{1}, \ldots, C_{p}$ be compact nonsingular algebraic curves in $\mathbb{R}^{n}$. Assume that

(i) $C_{i} \cap C_{j} \cap C_{k}=\emptyset$ whenever $i, j, k$ are distinct.

(ii) for every point $x$ in $C_{i} \cap C_{j}$, where $i \neq j$, the tangent spaces to $C_{i}$ and $C_{j}$ at $x$ are distinct.

Then every continuous map from $C=C_{1} \cup \ldots \cup C_{p}$ into $\mathbb{G}_{r, s}(\mathbb{R})$ can be approximated by regular maps in the compact-open topology.

Proof. Although a more direct proof is possible, the one below is probably the shortest. According to [3, Theorem 13.3.1], it sufficies to prove that every topological real vector bundle $\xi$ (of constant positive rank) on $C$ is isomorphic to an algebraic vector bundle. Since $\xi$ is isomorphic to the direct sum of a line bundle and a trivial bundle, we may assume that $\xi$ itself is a line bundle. Let $h: C \rightarrow \mathbb{P}^{d}(\mathbb{R})$ be a classifying map for $\xi$, that is, $h$ is continuous and $\xi$ is isomorphic to $h^{*} \gamma_{d}$, where $\gamma_{d}$ is the universal line bundle on $\mathbb{P}^{d}(\mathbb{R})$. Since $\gamma_{d}$ is an algebraic bundle, it is enough to show that $h$ can be approximated by regular maps. We proceed as follows. The union $S$ of all the intersections $C_{i} \cap C_{j}$, with $i \neq j$, is a finite set. By [7, Lemma 2.1], for each $i$, there is a regular map $f_{i}: C_{i} \rightarrow \mathbb{P}^{d}(\mathbb{R})$ arbitrarily close to $h \mid C_{i}$ and equal to $h$ on $C_{i} \cap S$. The map $f: C \rightarrow \mathbb{P}^{d}(\mathbb{R})$, defined by $f \mid C_{i}=f_{i}$ for $1 \leq i \leq p$, is continuous and close to $h$. Moreover, by [2, Lemma 3], $f$ is actually a regular map (here (i) and (ii) are used, along with the fact that $\mathbb{P}^{d}(\mathbb{R})$ can be regarded as an algebraic subset of $\mathbb{R}^{q}$ for some $q$ ). The proof is complete.

It is convenient to introduce the following terminology and notation. By convention, submanifolds will always be closed subsets of the ambient manifold. For any compact smooth manifold $M$, we denote by $[M]$ its fundamental class in $H_{m}(M, \mathbb{Z} / 2)$, where $m=\operatorname{dim} M$. If $N$ is an $n$-dimensional smooth submanifold of $M$, we write $[N]_{M}$ for the homology class in $H_{n}(M, \mathbb{Z} / 2)$ represented by $N$, that is, $[N]_{M}=i_{*}([N])$, where $i: N \hookrightarrow M$ is the inclusion map. By a smooth curve in $M$ we mean a smooth submanifold of $M$ of dimension 1 .

Proof of Theorem 1.1. We may assume that $M$ is a smooth submanifold of $\mathbb{R}^{n}$, where $n \geq 2 \operatorname{dim} M+1$. Let $C_{1}, \ldots, C_{p}$ be connected smooth curves in $M$, whose homology classes $\left[C_{1}\right]_{M}, \ldots,\left[C_{p}\right]_{M}$ generate $G$. We choose these curves in general position in $M$. Morover, we can select them in such a way that

$$
e_{*}\left(H_{1}(C, \mathbb{Z} / 2)\right) \text { is generated by }\left[C_{1}\right]_{M}, \ldots,\left[C_{p}\right]_{M},
$$

where

$$
C=C_{1} \cup \ldots \cup C_{p}
$$


and $e: C \hookrightarrow M$ is the inclusion map. Indeed, condition $\left(a_{0}\right)$ can be easily arranged if $\operatorname{dim} M=2$, while for $\operatorname{dim} M \geq 3$ is is automatically satisfied since then $C_{1}, \ldots, C_{p}$ are pairwise disjoint (being in general position).

There is a smooth diffeomorphism $\theta: \mathbb{R}^{n} \rightarrow \mathbb{R}^{n}$ such that $\theta\left(C_{i}\right)$ is a nonsingular algebraic curve in $\mathbb{R}^{n}$ of genus $g$ for $1 \leq i \leq p$. Hence we may assume that

$\left(a_{1}\right) \quad C_{i}$ itself is a nonsingular algebraic curve in $\mathbb{R}^{n}$ of genus $g$ for $1 \leq i \leq p$.

Set

$$
\Gamma=\left\{v \in H^{1}(M, \mathbb{Z} / 2) \mid\langle v, \alpha\rangle=0 \text { for all } \alpha \in G\right\},
$$

where $\langle-,-\rangle$ is the scalar product (Kronecker index). Note that

$$
G=\left\{\alpha \in H_{1}(M, \mathbb{Z} / 2) \mid\langle v, \alpha\rangle=0 \text { for all } v \in \Gamma\right\} .
$$

By Lemma 2.1, for any positive integer $d$, there is an algebraic model $A$ of $\mathbb{P}^{d}(\mathbb{R})$ such that for every real algebraic curve $D$ of genus at most $g$, every rational map from $D$ into $A$ is constant. If $\Gamma$ is generated by $q$ elements, $B=A \times \cdots \times A$ is the $q$-fold product, and $d$ is sufficiently large (depending on $M$ ), then there is a smooth map

$$
h: M \rightarrow B
$$

with $\Gamma=h^{*}\left(H^{1}(B, \mathbb{Z} / 2)\right)$. Hence

$$
G=\left\{\alpha \in H_{1}(M, \mathbb{Z} / 2) \mid\left\langle h^{*}(b), \alpha\right\rangle=0 \text { for all } b \in H^{1}(B, \mathbb{Z} / 2)\right\},
$$

and since $\left\langle h^{*}(b), \alpha\right\rangle=\left\langle b, h_{*}(\alpha)\right\rangle$, we get

$$
G=\left\{\alpha \in H_{1}(M, \mathbb{Z} / 2) \mid h_{*}(\alpha)=0\right\} .
$$

Assume $d \geq 2$. Since each homology class $\left[C_{i}\right]_{M}$ is in $G$, we get $\left(h \mid C_{i}\right)_{*}\left(\left[C_{i}\right]\right)=$ $h_{*}\left(\left[C_{i}\right]_{M}\right)=0$, and hence in view of $\left(a_{0}\right)$, the homomorphism

$$
(h \mid C)_{*}: H_{1}(C, \mathbb{Z} / 2) \rightarrow H_{1}(B, \mathbb{Z} / 2)
$$

is zero. This implies that the restriction map $h \mid C: C \rightarrow B$ is null homotopic since $H_{1}(B, \mathbb{Z} / 2)$ can be identified with the fundamental group of $B$. Actually, the map $h$ can be chosen in such a way that

$$
h: M \rightarrow B \text { is constant on } C .
$$

Indeed, let $K$ be a compact subset of $M$ such that $C$ is contained in the interior of $K$ and is a deformation retract of $K$, while $(M, K)$ is a polyhedral pair. Then $h \mid K: K \rightarrow B$ is null homotopic, and hence the homotopy extension theorem [10, p. 118, Corollary 5] implies the existence of a continuous map $h^{\prime}: M \rightarrow B$ homotopic to $h$ and constant on $K$. Thus there is a smooth map $h^{\prime \prime}: M \rightarrow B$ homotopic to $h^{\prime}$ and equal to $h^{\prime}$ on $C$. Replacing $h$ by $h^{\prime \prime}$, we may assume that $\left(a_{3}\right)$ is satisfied.

Henceforth assume $d$ is even. This implies that $A$, being an algebraic model of $\mathbb{P}^{d}(\mathbb{R})$, has totally algebraic homology (see Section 1). Consequently, by Künneth's theorem,

$B$ has totally algebraic homology.

In view of $\left(a_{1}\right),\left(a_{3}\right),\left(a_{4}\right)$, and Lemma 2.2, we can apply [2, Theorem 3] (cf. also [1, Proposition 2.8]) to $M, C, h: M \rightarrow B$. Therefore there exist a nonsingular algebraic subset $X$ of $\mathbb{R}^{n}$, a smooth diffeomorphism $\varphi: M \rightarrow X$, and a regular map 
$f: X \rightarrow B$ such that $C \subset X, \varphi(x)=x$ for all $x$ in $C$, and $f \circ \varphi$ is homotopic to $h$. In particular, $\varphi_{*}\left(\left[C_{i}\right]_{M}\right)=\left[C_{i}\right]_{X}$ for $1 \leq i \leq p$, which implies

$$
\varphi_{*}(G) \subseteq A(X, g) \subseteq B(X, g) .
$$

Moreover, making use of $\left(a_{2}\right)$, we get

$$
\varphi_{*}(G)=\left\{\beta \in H_{1}(X, \mathbb{Z} / 2) \mid f_{*}(\beta)=0\right\} .
$$

It remains to prove that if $\delta$ is a homology class in $B(X, g)$ represented by an algebraic curve $D$ in $X$ of genus at most $g$, then $\delta$ is in $\varphi_{*}(G)$, or equivalently, $f_{*}(\delta)=0$. The last equality holds, the restriction map $f \mid D: D \rightarrow B$ being constant (see how $B$ is constructed). The proof is complete.

Proof of Theorem 1.2. We may assume that $M$ is a smooth submanifold of $\mathbb{R}^{n}$, where $n \geq 2 \operatorname{dim} M+1$. Let $C_{1}, \ldots, C_{p}$ be connected smooth curves in $M$, whose homology classes $\left[C_{1}\right]_{M}, \ldots,\left[C_{p}\right]_{M}$ generate $G$. We choose these curves in general position in $M$, and so that

$$
e_{*}\left(H_{1}(C, \mathbb{Z})\right) \text { is generated by } o_{C_{1}}, \ldots, o_{C_{p}},
$$

where

$$
C=C_{1} \cup \ldots \cup C_{p},
$$

$e: C \hookrightarrow M$ is the inclusion map, and $o_{C_{i}}$ is the homology class in $H_{1}(M, \mathbb{Z})$ represented by $C_{i}$ endowed with some orientation. Furthermore, as in the proof of Theorem 1.1 , we may assume that

$$
C_{i} \text { is a nonsingular algebraic curve in } \mathbb{R}^{n} \text { of genus } 0 \text { for } 1 \leq i \leq p \text {. }
$$

Set

$$
\Gamma=\left\{v \in H^{1}(M, \mathbb{Z} / 2) \mid\langle v, \alpha\rangle=0 \text { for all } \alpha \in G\right\},
$$

and let $v_{1}, \ldots, v_{q}$ be generators of $\Gamma$.

Since $H_{1}(M, \mathbb{Z})$ has no 2-torsion, the group $H^{2}(M, \mathbb{Z})$ has no 2-torsion, which in turn implies that the restriction modulo 2 homomorphism $H^{1}(M, \mathbb{Z}) \rightarrow H^{1}(M, \mathbb{Z} / 2)$ is surjective (the universal coefficient theorem). Hence, in view of the orientability of $M$, the homology class Poincaré dual to $v_{j}$ can be represented by a codimension 1 orientable smooth submanifold $N_{j}$ of $M$. If $N_{j}$ is disconnected, then joining two connected components of $N_{j}$ with a tube in $M$, without modifying the remaining components, we obtain an orientable smooth submanifold $N_{j}^{\prime}$ of $M$ with $\left[N_{j}^{\prime}\right]_{M}=\left[N_{j}\right]_{M}$ (orientability of $N_{j}^{\prime}$ is not a problem here since we do not preassign orientations to the connected components of $N_{j}$, and we join with a tube only two connected components). After performing such an operation finitely many times, we may assume that $N_{j}$ is connected for $1 \leq j \leq q$. Moreover, we choose $N_{1}, \ldots, N_{q}$ so that $C_{1}, \ldots, C_{p}$, $N_{1}, \ldots, N_{q}$ are in general position in $M$.

Let $E$ be a complex elliptic curve defined over $\mathbb{R}$, whose set of real points $A=E(\mathbb{R})$ is connected. In particular, $A$ is diffeomorphic to the unit circle. According to the classical Pontryagin-Thom construction, there exist a smooth map $h_{j}: M \rightarrow A$ and a regular value $y_{j} \in A$ of $h_{j}$ with $N_{j}=h_{j}^{-1}\left(y_{j}\right)$, cf. [8]. Consequently,

$$
v_{j}=h_{j}^{*}(w),
$$


where $w$ is the unique generator of $H^{1}(A, \mathbb{Z} / 2) \cong \mathbb{Z} / 2$ (cf. [6, Proposition 2.15] if desired). We assert that each restriction map $h_{j} \mid C_{i}: C_{i} \rightarrow A$ is null homotopic for $1 \leq i \leq p$ and $1 \leq j \leq q$. Indeed, we have

$$
\begin{aligned}
\left\langle w,\left(h_{j} \mid C_{i}\right)_{*}\left(\left[C_{i}\right]\right)\right\rangle & =\left\langle w, h_{j_{*}}\left(\left[C_{i}\right]_{M}\right)\right\rangle \\
& =\left\langle h_{j}^{*}(w),\left[C_{i}\right]_{M}\right\rangle \\
& =\left\langle v_{j},\left[C_{i}\right]_{M}\right\rangle \\
& =0,
\end{aligned}
$$

where the last equality follows from the definition of $\Gamma$. Thus $\left(h_{j} \mid C_{i}\right)_{*}\left(\left[C_{i}\right]\right)=0$, which means that the topological degree modulo 2 of $h_{j} \mid C_{i}$ is 0 . Since $C_{i}$ is transverse to $N_{j}=h_{j}^{-1}\left(y_{j}\right)$ in $M$, the point $y_{j}$ is a regular value of $h_{j} \mid C_{i}$, and hence the set

$$
\left(h_{j} \mid C_{i}\right)^{-1}\left(y_{j}\right)=C_{i} \cap N_{j}
$$

consists of an even number of points, say, $m_{i j}$ of them. If orientations of $M, C_{i}, N_{j}$ are fixed, the topological degree of $h_{j} \mid C_{i}$ is equal, up to sign, to the intersection number $n_{i j}$ of $C_{i}$ and $N_{j}$ in $M$. Since $C_{i}$ and $N_{j}$ are connected, and $m_{i j}$ is even, it follows that $n_{i j}=0$. Hence $h_{j} \mid C_{i}$ is null homotopic, as asserted. Consequently, in view of $\left(b_{0}\right)$, the homomorphism

$$
\left(h_{j} \mid C\right)_{*}: H_{1}(C, \mathbb{Z}) \rightarrow H_{1}(A, \mathbb{Z})
$$

is zero. This implies that the restriction map $h_{j} \mid C: C \rightarrow A$ is null homotopic, since $H_{1}(A, \mathbb{Z})$ can be identified with the fundamental group of $A$.

Let $B=A \times \cdots \times A$ be the $q$-fold product. The smooth map

$$
h=\left(h_{1}, \ldots, h_{q}\right): M \rightarrow B
$$

satisfies

$$
\Gamma=h^{*}\left(H^{1}(B, \mathbb{Z} / 2)\right) .
$$

Moreover, the restriction map $h \mid C: C \rightarrow B$ is null homotopic. Now, arguing as in the proof of Theorem 1.1, we obtain

$$
G=\left\{\alpha \in H_{1}(M, \mathbb{Z} / 2) \mid h_{*}(\alpha)=0\right\} .
$$

Also, modifying $h$ without affecting $\left(b_{2}\right)$, we may assume

$$
h: M \rightarrow B \text { is constant on } C .
$$

Since $A$ is a compact connected nonsingular real algebraic curve,

$$
B \text { has totally algebraic homology. }
$$

The next step is again as in the proof of Theorem 1.1. In virtue of $\left(b_{1}\right),\left(b_{3}\right),\left(b_{4}\right)$ and Lemma 2.2, there exist a nonsingular algebraic subset $X$ of $\mathbb{R}^{n}$, a smooth diffeomorphism $\varphi: M \rightarrow X$, and a regular map $f: X \rightarrow B$ such that $C \subset X, \varphi(x)=x$ for all $x$ in $C$, and $f \circ \varphi$ is homotopic to $h$. This in turn implies

$$
\varphi_{*}(G) \subseteq A(X, 0) \subseteq D(X),
$$

and combined with $\left(b_{2}\right)$ yields

$$
\varphi_{*}(G)=\left\{\beta \in H_{1}(X, \mathbb{Z} / 2) \mid f_{*}(\beta)=0\right\} .
$$

It remains to prove that if $\delta$ is a homology class in $D(X)$ represented by a dividing algebraic curve $D$ in $X$, then $\delta$ is in $\varphi_{*}(G)$, or equivalently, $f_{*}(\delta)=0$. To this end, 
choose a compact nonsingular real algebraic curve $\tilde{D}$ and a regular map $\pi: \tilde{D} \rightarrow$ $D$ such that $\pi$ is a birational isomorphism. In particular, $\tilde{D}$ is a dividing curve. Moreover, we have

$$
f_{*}(\delta)=(f \circ e \circ \pi)_{*}([\tilde{D}])=0,
$$

where $e: D \hookrightarrow X$ is the inclusion map, and the last equality is a consequence of $[5$, Theorem 1.1]. The proof is complete.

\section{Acknowledgements}

The paper was completed at the Max-Planck-Institut für Mathematik in Bonn, whose support and hospitality are gratefully acknowledged.

\section{References}

[1] S. Akbulut and H. King, The topology of real algebraic sets with isolated singularities, Ann. of Math. 113(1981), 425-446.

[2] R. Benedetti and A. Tognoli, Approximation theorems in real algebraic geometry, Boll. Unione Mat. Ital., Suppl. 2(1980), 198-211.

[3] J. Bochnak, M. Coste and M.-F. Roy, Real Algebraic Geometry, Ergebnisse der Math. und ihrer Grenzgeb. Folge 3, Vol. 36, Berlin Heidelberg New York, Springer, 1998.

[4] J. Bochnak and W. Kucharz, On homology classes represented by real algebraic varieties, Banach Center Publications Vol. 44, 21-35, Warsaw, 1998.

[5] J. Bochnak and W. Kucharz, Complexification of real algebraic varieties and vanishing of homology classes, Bull. London Math. Soc. 33(2001), 32-40.

[6] A. Borel et A. Haefliger, La classe d'homologie fondamentale d'un espace analytique, Bull. Soc. Math. France 89(1961), 461-513.

[7] W. Kucharz, Algebraic morphisms into rational real algebraic surfaces, J. Algebraic Geometry 8(1999), 569-579.

[8] J. Milnor, Topology from the Differentiable Viewpoint, University of Virginia Press, 1966.

[9] J. Nash, Real algebraic manifolds, Ann. of Math. 56(1952), 405-421.

[10] E. Spanier, Algebraic Topology, New York Berlin Heidelberg, Springer.

[11] A. Tognoli, Su una congettura di Nash, Ann. Scuola Norm. Sup. Pisa Sci. Fis. Mat. (3) 27(1973), 167-185.

Department of Mathematics and Statistics, University of New Mexico, MSC03 2150, Albuquerque, New Mexico 87131-0001, U.S.A.

E-mail address: kucharz@math.unm.edu 